\title{
Resección de metástasis pulmonares*
}

\author{
Drs. GONZALO CARDEMIL H. ${ }^{1}$, ANDRÉS MARAMBIO G. ${ }^{1}$, JORGE SALGUERO A. ${ }^{1}$, \\ GONZALO FERNÁNDEZ R. ${ }^{1}$, MAURICIO DÍAZ B. ${ }^{1}$, Ints. CATALINA JARPA M. ${ }^{2}$, \\ PAULINA DÍAZ V. ${ }^{2}$, RODRIGO DÍAZ P. ${ }^{2}$, JUAN P. MARAMBIO G. ${ }^{2}$
}

\author{
Departamento de Cirugía Hospital Clínico Universidad de Chile. \\ 2 Internos de Medicina. Facultad de Medicina. Universidad de Chile. \\ Santiago, Chile.
}

\begin{abstract}
\section{Resection of lung metastases}

Introduction: Surgical treatment of lung metastases (LM) is a therapy used for several types of metastatic cancers. However, the type of surgery and its outcome is controversial. Aim: To describe a series of patients with LM undergoing surgical treatment, their results and long-term follow up. Material and Methods: We retrospectively reviewed all the medical records of patients with the diagnosis of LM, of whatever origin, operated at our hospital between the years 2002 and 2008. We analyzed their clinical characteristics, treatment, morbidity, mortality and results. Results: During this period 24 patients with LM were operated. The mean age was $52.8 \pm 16.6$ years. Most patients had kidney cancer as primary tumour. The usual clinical presentation was multiple bilateral nodules. The surgical approach was in general a video-thoracoscopy (40.9\%) associated with a wedge resection of the lesions $(81.8 \%)$. In $83.4 \%$ of cases, there were no post-operative complications. Seventy six percent of the patients relapse, in an average of $16.7 \pm 12$ months and $68.7 \%$ were reoperated for a new resection. The probability of surviving more than 3 and 5 years post metastasectomy was $57 \%$ and $36 \%$ respectively. Conclusions: In this series the LM were mostly secondary to renal cancer. The video-thoracoscopy is an emergent and safe technique in the treatment of LM, being the main type of surgical approach in our patients. Despite the high percentage of reoperations, the survival rate is comparable to the rest of the published series.
\end{abstract}

Key words: Lung metastases, metastasectomy.

\section{Resumen}

Introducción: El tratamiento quirúrgico de las metástasis pulmonares (MP) constituye una terapia usada para varios tipos de cánceres metastásicos. Sin embargo, el tipo de cirugía y sus resultados es controversial, por lo que hemos decidido estudiarla. Objetivo: Describir clínicamente una serie de pacientes con MP sometidos a tratamiento quirúrgico, sus resultados y seguimiento a largo plazo. Material y Método: Se revisaron retrospectivamente las fichas de pacientes con el diagnóstico de MP, de cualquier origen, operados en nuestro hospital entre los años 2002 y 2008. Se analizaron las características clínicas, tratamiento, morbi-

*Recibido el 15 de Agosto de 2009 y aceptado para publicación el 20 de Marzo de 2010.

Correspondencia: Dr. Andrés Marambio G.

Santos Dumont 999, Santiago, Chile.

Código Postal: 8380456. Fax: 56-2-7370844.

E-mail: amarambio@med.uchile.cl 
mortalidad y resultados. Resultados: Durante este período se operaron 24 pacientes por MP. El promedio de edad fue 52,8 $\pm 16,6$ años. La mayoría de los pacientes tenía un cáncer renal como tumor primario. La forma habitual de presentación fueron múltiples nódulos bilaterales. El abordaje quirúrgico más frecuente fue por videotoracoscopía (40,9\%) asociado a una resección en cuña de las lesiones $(81,8 \%)$. En el $83,4 \%$ no hubo complicaciones post operatorias. El 76,4\% de los pacientes recidivó, en promedio a los $16,7 \pm 12$ meses y el $68,7 \%$ fue reintervenido para una nueva resección. La probabilidad de sobrevivir más de 3 y 5 años post metastasectomía fue de 57\% y 36\% respectivamente. Conclusiones: En esta serie las MP fueron principalmente secundarias a cáncer renal. La videotoracoscopía es una cirugía emergente y segura en el tratamiento de las MP, siendo la principal forma de abordaje quirúrgico en nuestros pacientes. A pesar del elevado porcentaje de reintervenciones, la sobrevida es comparable al resto de las series publicadas.

Palabras clave: Metástasis pulmonares, metastasectomía.

\section{Introducción}

Antes los pacientes con MP, sea cualquiera su origen, se consideraban fuera del alcance quirúrgico. Hace más de un siglo surge el primer reporte sobre resección de MP en un paciente con un sarcoma de pared costal ${ }^{1} \mathrm{y}$ en 1947 se reporta la primera serie de pacientes ${ }^{2}$. Los autores, Alexander y Haight, establecen los criterios básicos a cumplir antes de realizar cualquier metastasectomía pulmonar. Estos criterios han sido modificados con el tiempo y se refieren a: 1) el tumor primario debe estar controlado; 2) posibilidad de resecar todas las metástasis; 3) no debe haber evidencia de enfermedad extrapulmonar (o enfermedad extrapulmonar controlable según algunos autores); 4) status cardio-pulmonar del paciente suficiente para compensar la resección y 5) ausencia de alguna mejor terapia. Esto se aplica a cualquier tumor que metastize en el pulmón, aunque algunos cánceres como el osteosarcoma, sarcomas de partes blandas, carcinoma de células renales (CCR) y los cánceres de cabeza y cuello, tienen preferencia por este órgano y una mayor resecabilidad, siendo más susceptibles de una metastasectomía pulmonar ${ }^{3}$. Siguiendo estos criterios se han obtenido en forma global sobrevidas a 5 años que están entre el $36-48 \% 0^{4,5}$ y hoy en día estas indicaciones se han ampliado, aplicándose incluso en presencia de recidivas extrapulmonares concomitantes. Son pacientes ultraseleccionados representando un $15-25 \%$ del to$\mathrm{tal}^{6}$. La literatura muestra resultados contradictorios, sobretodo al evaluar factores pronósticos que nos permitan predecir qué pacientes serán beneficiados. Debido a esto es que no existen pautas absolutas y la conducta quirúrgica varía ampliamente entre un centro y otro.

Nuestro objetivo es describir una serie de pacientes sometidos a metastasectomía pulmonar en el Hospital Clínico de la Universidad de Chile (HCUCH), los tipos de cáncer involucrados, las técnicas quirúrgicas utilizadas, sus resultados y evolución a largo plazo.

\section{Material y Método}

El presente estudio fue diseñado como retrospectivo y descriptivo de los casos de MP, de cualquier origen, sometidos a metastasectomía entre los años 2002 y mediados de 2008 por el equipo de cirugía de tórax del HCUCH. Se recopilaron las fichas de todos los pacientes con el diagnóstico de "resección de MP" y se recolectaron los datos concernientes a los antecedentes médicos, tipo de tumor primario, tratamiento empleado y fecha, presentación clínica de las MP y fecha, exámenes de imágenes, tipo de cirugía realizada para la metastasectomía, resultado histopatológico, tratamientos coadyuvantes, seguimiento clínico y de imágenes, recidivas, reintervenciones y sobrevida hasta el año 2008. Para esto último se realizó seguimiento telefónico y se consultó en el Registro Civil por la eventual defunción de los pacientes que no pudieron ser contactados. Se incluyeron sólo pacientes con MP secundarias a tumores extrapulmonares, excluyéndose los cánceres primarios de pulmón y pleura. Se consideró videotoracoscopía (VTC) como el procedimiento en que se accede a la cavidad torácica sólo mediante trócares y VATS (video-assisted thoracoscopic surgery) cuando además se realiza una incisión entre 6 y $8 \mathrm{~cm}$ para apoyo manual. La elección del método de abordaje fue según la preferencia del cirujano. Se definió persistencia de la enfermedad como la pesquisa de lesiones pulmonares durante los dos meses siguientes a la cirugía, mientras que recidiva cuando aparecieron posteriormente. Para el análisis de sobrevida se utilizó el método de Kaplan-Meier.

\section{Resultados}

Durante el período de estudio se operaron 24 pacientes, 12 fueron hombres. El promedio de edad fue 52,8 $\pm 16,6$ años (rango 17-80, mediana 56) y habitualmente el compromiso pulmonar se presentó como un hallazgo. En una menor proporción $(25 \%)$ 
Tabla 1. Características clínicas

\begin{tabular}{lc}
\hline Variable & n pacientes \\
\hline Número de pacientes & 24 \\
Edad promedio (años) & $52,8 \pm 16,6$ \\
Género (Hombre/Mujer) & $12 / 12$ \\
Presentación clínica MP: & \\
Hallazgo & 18 \\
Tos seca & 3 \\
Disnea & 1 \\
Dolor & 1 \\
Neumotórax & 1 \\
Tumor Primario: & \\
Renal & 11 \\
Colon & 4 \\
Células germinales & 2 \\
Cabeza y cuello & 2 \\
Recto & 1 \\
Osteosarcoma & 1 \\
Sarcoma de partes blandas & 1 \\
Carcinoide & 1 \\
Condrosarcoma & 1 \\
\hline
\end{tabular}

hubo síntomas que orientaron al estudio pulmonar (tos seca en 3 casos, disnea, dolor y neumotórax espontáneo en 1 caso cada uno). La mayoría de los pacientes tenía un CCR como tumor primario (11), luego de colon (4) y finalmente de células germinales (teratoma) o de cabeza y cuello ( 2 casos cada uno). El resto de los casos correspondía a cáncer de recto, osteosarcoma, sarcoma de partes blandas (tímico), carcinoide y condrosarcoma, todos con un caso cada uno (Tabla 1). En 21 pacientes $(87,5 \%)$ las MP fueron diagnosticadas dentro del estudio de diseminación del tumor primario, en promedio 14,7 $\pm 25,4$ meses después del diagnóstico inicial (rango 0-78 meses, mediana 4). Los casos con mayor intervalo de tiempo entre el diagnóstico del tumor primario y el de las MP correspondieron a dos CCR (5 y 6,5 años) y un carcinoma adenoideo quístico de paladar (6 años). En sólo 3 casos el compromiso pulmonar fue el hallazgo inicial, siendo el tumor primario diagnosticado 2 meses después en dos casos y 4 meses después en el tercero. Se contaba con la Tomografía Axial Computada (TAC) preoperatoria de 22 pacientes, de las cuales $18(81,8 \%)$ evidenciaban compromiso bilateral. En 8 pacientes se informaba la presencia de "múltiples nódulos bilaterales". En los otros 14 pacientes variaba entre 0 y 7 nódulos en
Tabla 2. Técnica quirúrgica, tratamiento coadyuvante y complicaciones

\begin{tabular}{lc}
\hline Variable & n pacientes \\
\hline Vía de abordaje*: & 9 \\
VTC & 7 \\
Toracotomía & 6 \\
VATS & \\
Tipo de resección*: & 18 \\
Cuña & 3 \\
Segmentectomía & 1 \\
Lobectomía & \\
Complicaciones postoperatorias & 3 \\
Neumotórax & 1 \\
Neumomediastino & 1 \\
Enfisema subcutáneo & 1 \\
Fístula bronquio alveolar & 2 \\
Atelectasia & 1 \\
Hemorragia & 3 \\
Neumonia & 1 \\
TEP & 9 \\
Tratamiento coadyuvante & 3 \\
Quimioterapia & \\
Inmunoterapia & \\
\hline
\end{tabular}

* sobre un total de 22 pacientes.

el lado derecho (promedio de 2,3 con mediana de 2) y entre 0 y 14 en el izquierdo (promedio de 2,4 con mediana de 1). En 7 pacientes se describía la presencia de adenopatías mediastínicas.

Para el análisis de la cirugía se contaba con la información de 22 pacientes dado el extravío de 2 protocolos operatorios. En estos, el abordaje quirúrgico consistió en una VTC en 9 pacientes (40,9\%), la cual fue bilateral secuencial o escalonada con 4 semanas de diferencia entre ambos abordajes en 2 y 1 paciente respectivamente. A siete pacientes $(31,8 \%)$ se les realizó una toracotomía póstero-lateral, la cual fue bilateral en 4 casos, ya sea en forma secuencial o escalonada (1 paciente). A un paciente se le realizó una toracotomía derecha, a otro una toracotomía izquierda asociada a una VTC derecha en un mismo tiempo quirúrgico y a otro una toracotomía izquierda seguida de una VTC derecha 1 mes después. Al resto de los pacientes se les realizó una VATS, la cual fue bilateral en 4 casos (Tabla 2). La resección de las metástasis fue realizada mediante una incisión en cuña en 18 pacientes. En casos aislados se realizó segmentectomía, bilobectomía, combinación de segmentectomía más resección en cuña y segmentectomía más resección de cuña hepática, todos con un caso cada uno. Sólo se realizó biopsia ganglionar 
cuando las imágenes preoperatorias evidenciaban adenopatías. La biopsia rápida de las metástasis fue compatible con el tumor primario en todos los casos, a excepción de un paciente con un tumor de células germinales, en la cual reveló tejido necrótico e inflamatorio, pero la biopsia definitiva fue concordante con teratoma. En 12 pacientes se asoció a la metastasectomía tratamiento coadyuvante, ya sea con quimioterapia (donde se incluyen todos los pacientes con tumores de células germinales y de colon) y/o inmunoterapia (3 pacientes con CCR recibieron interferón). Cuatro pacientes $(16,6 \%)$ presentaron complicaciones postoperatorias. Un paciente sometido a una VTC bilateral escalonada presentó un neumotórax más un Tromboembolismo pulmonar (TEP) luego de la primera cirugía, y un sangrado del lecho operatorio luego de la segunda, que no requirió nueva cirugía. Este mismo paciente evolucionó con una neumonía durante esta última hospitalización. El segundo paciente presentó un neumotórax asociado a enfisema subcutáneo y una atelectasia del lóbulo inferior izquierdo (LII) luego de una VTC bilateral secuencial. El tercer paciente, luego de una toracotomía asociada a una VTC un mes después, presentó una neumonía luego de la última cirugía y el cuarto paciente presentó un neumotórax asociado a neumomediastino, una atelectasia del lóbulo inferior derecho (LID), una fístula bronquio alveolar y finalmente una neumonía luego de una VATS bilateral secuencial por una recidiva (Tabla 2).

Para el seguimiento se contaba con la información de 20 pacientes, de los cuales tres (15\%) presentaron persistencia de la enfermedad. El primer paciente presentaba metástasis secundarias a un osteosarcoma y fue sometido a una cirugía paliativa por neumotórax. El segundo paciente tenía una TAC preoperatoria que informaba múltiples metástasis pulmonares secundarias a un CCR. Fue sometido a una VATS bilateral resecándose 7 metástasis a izquierda y 5 a derecha. Dos meses después se realiza una nueva TAC que informa múltiples nódulos bilaterales. El tercer paciente tenía dos metástasis secundarias a un cáncer de colon siendo sometido a una VATS bilateral, sin embargo, la radiografía postoperatoria revela persistencia de imágenes. Posteriormente recibió quimioterapia, sin embargo, falleció a los 5 meses de operado. Del resto de los pacientes, $13(76,4 \%)$ recidivaron en promedio a los $16,7 \pm 12$ meses (rango 3-36, mediana 9), con la reaparición de nuevos nódulos pulmonares, masa pulmonar en dos pacientes y derrame pleural en uno. $\mathrm{Al}$ analizar este grupo de 16 pacientes (persistencia más recidiva) se obtiene que $11(68,7 \%)$ fueron reintervenidos al menos una vez para una nueva resección de las lesiones, habitualmente por una toracotomía
Tabla 3. Evolución de los pacientes

\begin{tabular}{lc}
\hline Variable & n pacientes \\
\hline Recidiva* & 13 \\
Pacientes & $16,7 \pm 12$ \\
Tiempo post $1^{\circ}$ cirugía & \\
$\quad$ (promedio en meses) & \\
Reintervenciones*: & 8 \\
Una vez & 2 \\
Dos veces & 1 \\
Tres veces & \\
Muerte: & 11 \\
Pacientes & $26 \pm 17,3$ \\
Tiempo post $1^{\circ}$ cirugía & \\
$\quad$ (promedio en meses) & \\
Sobrevida & \\
3 años & $57 \%$ \\
5 años & $36 \%$ \\
\hline
\end{tabular}

*sobre un total de 20 pacientes.

más resección en cuña, asociándose además terapia coadyuvante en 5 de ellos. Dos pacientes fueron sometidos a una tercera metastasectomía y uno a una cuarta (Tabla 3). Al finalizar este trabajo 11 pacientes $(45,8 \%)$ habían fallecido por progresión de su enfermedad, en promedio a los $26 \pm 17,3$ meses después de la primera metastasectomía, con un rango de sobrevida que va entre los 5 meses (osteosarcoma) y los 4 años (CCR). La probabilidad de sobrevivir más de 3 y 5 años después de esta cirugía fue de $57 \%$ y $36 \%$ respectivamente (Tabla 3 y Figura 1 ).

\section{Discusión}

Hemos querido revisar nuestra experiencia en la metastasectomía pulmonar y sus resultados, dado la inexistencia de publicaciones en el medio nacional. En el presente estudio destaca el CCR como el más frecuente, con el 45,8\% de nuestros pacientes, a diferencia de lo que ocurre en la mayoría de los estudios, en donde el cáncer colorectal suele ser el más común ${ }^{7}$. Este último, a pesar de tener baja afinidad por el pulmón como órgano de metástasis y que sólo el $1 \%$ de los pacientes cumple los criterios de resección, es muy prevalente, influyendo de esta forma en los resultados finales. En este cáncer también se ha visto beneficio con la resección concomitante de metástasis hepáticas ${ }^{3}$, lo que se reporta en uno de nuestros pacientes. El CCR tiene su principal sitio de metástasis en el pulmón y su respuesta a la 


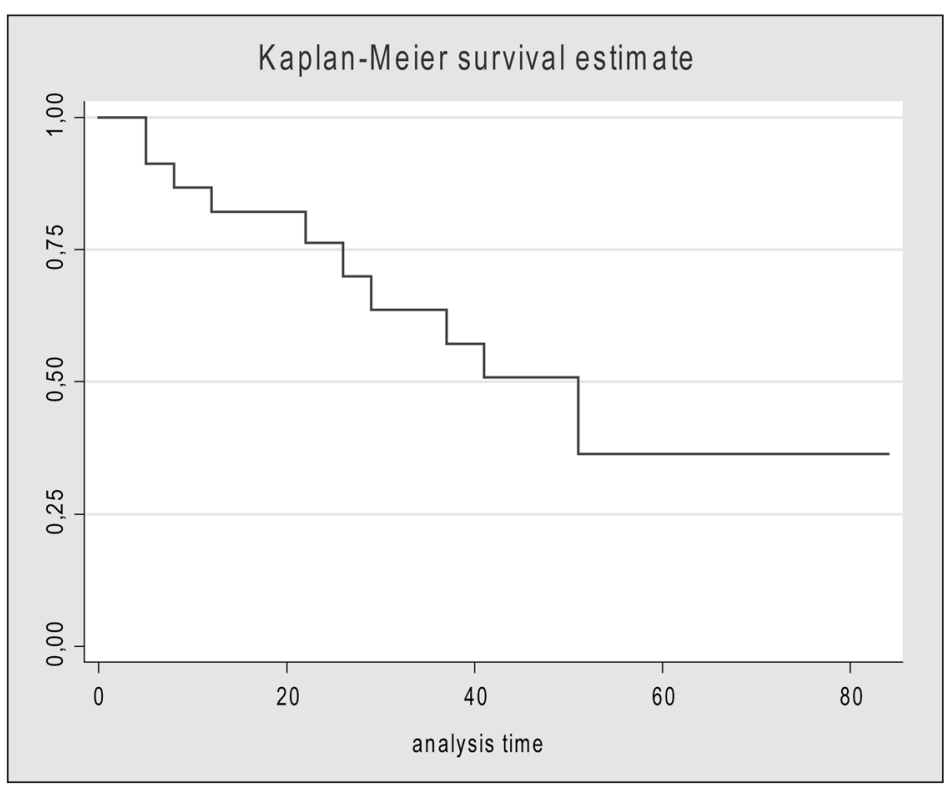

Figura 1. Probabilidad de sobrevida luego de metastasectomía en cohorte de pacientes (24) con MP operados en el HCUCH (tiempo en meses). quimioterapia, a diferencia del cáncer colorrectal, es menor al $10 \%$. Se ha visto que su estadío metastásico tiene una sobrevida de 6-12 meses $^{8}$ y que a 5 años la tasa de sobrevida con quimioterapia es cercana al $3 \%{ }^{9}$, mientras que grupos que además reciben cirugía esta se eleva a $37-45 \%{ }^{10,11}$. En nuestra serie, la probabilidad de sobrevivir más de 5 años en este subgrupo de pacientes alcanzó el $20 \%$. De esta forma la metastasectomía pasa a ser un tratamiento de primera línea en los CCR con MP, siempre que se cumplan los criterios ya enunciados. En otros cánceres, como los tumores de células germinales y el cáncer de mama, ocupa una segunda línea de tratamiento luego de la quimioterapia, con claro beneficio $^{12}$.

Hemos visto que las MP suelen ser asintomáticas y bilaterales, lo que concuerda con otros reportes, dado el inusual compromiso traqueobronquial o mediastínico ${ }^{6}$. Algunos autores recomiendan realizar una fibrobroncoscopía preoperatoria o incluso una mediastinoscopía, si se sospecha compromiso central, o al estar frente a metástasis de tumores que tienden a presentar compromiso endobronquial, como el cáncer de mama, colon o riñón ${ }^{13}$. Sin embargo, otros recomiendan realizar este procedimiento de rutina ${ }^{6,14}$. En nuestra serie sólo un paciente tenía una fibrobroncoscopía preoperatoria. Este tenía un CCR de base y presentaba un nódulo en el lóbulo inferior derecho en cercanía de los bronquios. La biopsia por esta vía reportó un adenocarcinoma, siendo interpretado como un tumor primario y realizándose una bilobectomía, de lóbulos medio e inferior derecho.
Nuestro abordaje más frecuente fue la VTC. Sobre cuál técnica es la ideal, hay gran controversia siendo un tema relevante para nuestros resultados ya que el $64,8 \%$ de todas las cirugías realizadas (incluyendo reintervenciones) fue por VTC o VATS. La mayoría de los cirujanos de tórax prefiere la toracotomía, dado que por la vía toracoscópica no se estaría cumpliendo el segundo criterio básico y único factor pronóstico validado universalmente, la resección de todas las lesiones ${ }^{15}$. Esto se basa en que las imágenes preoperatorias son fundamentales para programar la resección y se ha visto que la TAC subestima lesiones hasta en un $27-40 \%$ de las veces $^{15-17}$. Por esto habría que palpar directamente la superficie pulmonar buscando lesiones no diagnosticadas, e incluso en forma bilateral, a pesar que las imágenes nos revelen compromiso unilateral. Sin embargo, estos trabajos fueron realizados hace más de 10 años mientras que hoy contamos con nuevas generaciones de TAC helicoidal de alta resolución, lo que permite detectar lesiones más pequeñas. Al respecto, un estudio más reciente, revela que los nódulos pequeños no detectados por imágenes $(<$ $5 \mathrm{~mm}$ de diámetro) habitualmente son benignos por lo que la palpación tendría un rol limitado, recomendándose la $\mathrm{VTC}^{18}$. De la misma forma se ha reportado que la exploración bilateral es igual que la vía unilateral ${ }^{19,20}$. Al revisar nuestra serie hemos visto que los casos asociados a toracotomía correspondían a reintervenciones, presencia de lesiones centrales, preferencia del cirujano (un cirujano sólo realizó toracotomías) o imposibilidad técnica para lograr una adecuada anestesia monopulmonar. Por 
el momento la discusión no está zanjada, aunque la toracotomía continúa siendo la principal vía de abordaje de las MP en la mayoría de los centros ${ }^{4}$. Por último, comentar la revisión hecha por Pfannschmidt et $\mathrm{a}^{21}$, en la cual la vía de abordaje no fue un factor pronóstico. Desde el punto de vista del tipo de resección y tal como hemos visto en este trabajo, la incisión en cuña sigue siendo la más frecuente ${ }^{8,22-24}$, dado que las metástasis suelen ser periféricas por la mayor irrigación de esta zona. Se ha comprobado que procedimientos mayores como lobectomía o neumonectomía no mejoran los resultados ${ }^{13}$. También hemos visto que esta cirugía ofrece una tasa aceptable de complicaciones $(16,6 \%)$ y sin mortalidad relacionada al procedimiento en este estudio, la que se ha reportado cercana al $1 \%{ }^{4}$.

Al analizar la evolución vemos que un $76,4 \%$ recidivó, en promedio a los 16 meses, siendo necesarias nuevas reintervenciones. El registro internacional de MP con 5.206 casos $^{4}$, reportó en 1997 una recurrencia del $53 \%$ en el subgrupo de pacientes sometidos a resección completa de sus lesiones (88\% del total), en promedio a los 10 meses de operados y principalmente en pacientes con tumores sarcomatosos y melanomas, aunque en este último caso, en general extratorácicas. La mayoría de nuestros pacientes fue reintervenido siendo la VTC y la toracotomía las vías de abordaje preferidas $(50 \%$ cada una). Las resecciones de recurrencias también han demostrado mejorar la sobrevida, sobre todo en sarcomas de tejidos blandos ${ }^{25}$. De hecho en el grupo de Pastorino ${ }^{4}$, la segunda metastasectomía se asoció a mejor pronóstico que la primera, lo que no ocurrió en esta serie, sin embargo, la sobrevida global fue similar a la nuestra ya que reportaron un $34 \%$ a 5 años de seguimiento.

Hemos visto que la resección de MP es útil y segura en pacientes seleccionados, pero que a pesar de su mayor aceptación y uso, aún existen muchas áreas de incertidumbre, tales como la vía de abordaje, la necesidad de disección linfonodal, los factores pronósticos preoperatorios.

\section{Referencias}

1. Weinlechner JW. Tumoren an der brustwand und deren behnadlung resection der rippeneroffnung der brusthohle und partielle entfernung der lunge. Wein Med Wochenschr 1882; 32: 589-591.

2. Alexander J, Haight C. Pulmonary resection for solitary metastatic sarcomas carcinomas. Surg Gynecol Obstet 1947; 85: 129-146.

3. Sternberg DI, Sonett JR. Surgical therapy of lung metastases. Semin Oncol 2007; 34: 186-196.

4. Pastorino U, Buyse M, Friedel G, Ginsberg RJ, Girard
P, Goldstraw P, et al. Long-term results of lung metastasectomy: prognostic analyses based on 5.206 cases. J Thorac Cardiovasc Surg 1997; 113: 37-49.

5. Robert JH, Ambrogi V, Mermillod B, Dahabreh D, Goldstraw P. Factors influencing long-term survival after lung metastasectomy. Ann Thorac Surg 1997; 63: 777-784.

6. Greelish JP, Friedberg JS. Secondary pulmonary malignancy. Surg Clin North Am 2000; 80: 633-657.

7. Tanaka Y, Maniwa Y, Nishio W, Yoshimura M, Okita Y. The optimal timing to resect pulmonary metastasis. Eur J Cardiothorac Surg 2008; 33: 1135-1138.

8. Yoneda K, Louie S, Shelton DK. Approach to pulmonary metastases. Curr Opin Pulm Med 2000; 6: 356 363.

9. Cerfolio RJ, Allen MS, Deschamps C, Daly RC, Wallrichs SL, Trastek VF, et al. Pulmonary resection of metastatic renal cell carcinoma. Ann Thorac Surg 1994; 57: 339-344.

10. Assouad J, Petkova B, Berna P, Dujon A, Foucault C, Riquet M. Renal cell carcinoma lung metastases surgery: pathologic findings and prognostic factors. Ann Thorac Surg 2007; 84: 1114-1120.

11. Murthy SC, Kim K, Rice TW, Rajeswaran J, Bukowski $\mathrm{R}$, DeCamp MM, et al. Can we predict long-term survival after pulmonary metastasectomy for renal cell carcinoma? Ann Thorac Surg 2005; 79: 996-1003.

12. Tanaka F, Li M, Hanaoka N, Bando T, Fukuse T, Hasegawa S, et al. Surgery for pulmonary nodules in breast cancer patients. Ann Thorac Surg 2005; 79: 1711-1714.

13. Rusch VW. Pulmonary metastasectomy. Chest 1995; 107: 322S-332S.

14. Quiros RM, Scott WJ. Surgical treatment of metastatic disease to the lung. Semin Oncol 2008; 35: 134-146.

15. McCormack PM, Bains MS, Beqq CB, Burt ME, Downey RJ, Panicek DM, et al. Role of video-assisted thoracic surgery in the treatment of pulmonary metastases: results of a prospective trial. Ann Thorac Surg 1996; 62: 213-216.

16. Swoboda L, Toomes H. Results of surgical treatment for pulmonary metastases. Thorac Cardiovasc Surg 1986; 34: 149-152.

17. Friedmann G, Bohndorf K, Krüger J. Radiology of pulmonary metastases: comparison of imaging techniques with operative findings. Thorac Cardiovasc Surg 1986; 34: 120-124.

18. Nakajima J, Murakawa T, Fukami T, Sano A, Sugiura $\mathrm{M}$, Takamoto S. Is finger palpation at operation indispensable for pulmonary metastasectomy in colorectal cancer? Ann Thorac Surg 2007; 84: 16801684.

19. Roth JA, Pass HI, Wesley MN, White D, Putnam JB, Seipp C. Comparison of median sternotomy and thoracotomy for resection of pulmonary metastases in patients with adult soft-tissue sarcomas. Ann Thorac 
Surg 1986; 42: 134-138.

20. Younes RN, Gross JL, Deheinzelin D. Surgical resection of unilateral metastases: is bilateral thoracotomy necessary? World J Surg 2002; 26: 1112-1116.

21. Pfannschmidt J, Dienemann H, Hoffmann H. Surgical resection of pulmonary metastases from colorectal cancer: a systematic review of published series. Ann Thorac Surg 2007; 84: 324-338.

22. De la Haba JR, Algar J, Méndez MJ, Barreto I, Álvarez A, López J, et al. Surgical treatment of pulmonary metastases: experience with 40 patients. Eur J Surg Oncol 2002; 28: 49-54.
23. Kondo H, Okumura T, Ohde Y, Nakagawa K. Surgical treatment for metastatic malignancies. Pulmonary metastasis: indications and outcomes. Int J Clin Oncol 2005; 10: 81-85.

24. Lin JC, Wiechmann RJ, Szwerc MF, Hazelrigg SR, Ferson PF, Naunheìm KS, et al. Diagnostic and therapeutic video-assisted thoracic surgery resection of pulmonary metastases. Surgery 1999; 126: 636-642.

25. Rizzoni WE, Pass HI, Wesley MN, Rosenberg SA, Roth JA. Resection of recurrent pulmonary metastases in patients with soft-tissue sarcomas. Arch Surg 1986; 121: $1248-1252$. 